\title{
La ventilation non invasive en mode NAVA (neurally adjusted ventilatory assist) en réanimation pédiatrique
}

\author{
Non invasive ventilation with neurally adjusted ventilatory assist in the pediatric intensive \\ care unit
}

\author{
L. Ducharme-Crevier $\cdot$ S. Essouri $\cdot$ A. Larouche $\cdot$ G. Emeriaud \\ Reçu le 23 octobre 2013 ; accepté le 4 janvier 2014 \\ (C) SRLF et Springer-Verlag France 2014
}

Résumé La ventilation non invasive (VNI) est un outil thérapeutique utilisé dans la majorité des réanimations pédiatriques. L'objectif de la VNI est d'éviter le recours à l'intubation, mais près de $25 \%$ des enfants nécessitent finalement une ventilation invasive. La mauvaise synchronisation patientventilateur est un des facteurs d'échec de la VNI. Le récent mode NAVA (neurally adjusted ventilatory assist) permet d'améliorer la synchronisation et pourrait permettre d'améliorer l'efficacité de la VNI. L'objectif de cette revue est de faire le point sur les données actuelles de la littérature concernant le mode VNI-NAVA chez l'enfant et de fournir des repères pratiques pour l'utilisation de ce mode, à partir de ces évidences et de notre expérience clinique. Les études sur la VNI-NAVA sont essentiellement d'ordre physiologique et de courte durée. Elles confirment toutes l'amélioration de la synchronisation patient-ventilateur. Si l'expérience clinique suggère que certains patients bénéficient de cette optimisation du support, des études supplémentaires sont nécessaires pour évaluer l'impact clinique de la VNI-NAVA sur le confort, le taux d'échec et la durée du support ventilatoire des enfants de réanimation pédiatrique.

Mots clés Ventilation non invasive $\cdot$ Neurally adjusted ventilatory assist $\cdot$ Réanimation pédiatrique $\cdot$ Ventilation mécanique

Abstract Non invasive ventilation (NIV) is a therapeutic modality frequently used in pediatric intensive care units in

\footnotetext{
L. Ducharme-Crevier 'S. Essouri - A. Larouche ·

G. Emeriaud $(\bowtie)$

Soins intensifs pédiatriques, CHU Sainte-Justine,

Université de Montréal, 3175 chemin de la côte Sainte-Catherine,

Montréal (Qc) H3T 1C5, Canada

e-mail : guillaume.emeriaud@umontreal.ca

S. Essouri

Soins intensifs pédiatriques, CHU Kremlin Bicêtre,

Université Paris Sud, Le Kremlin Bicêtre, France
}

order to avoid intubation. However, about $25 \%$ of children fail and require invasive ventilation. Poor patient-ventilator synchrony is one of the reasons why patients fail to respond to NIV. The new mode of ventilation NAVA (neurally adjusted ventilatory assist) allows better patient-ventilator synchrony which could potentially improve the efficiency of NIV. The objectives of this paper are to review the available data, and propose practice guidelines on the use of NIVNAVA in critically ill children, based on published evidence and on our clinical experience. The available studies evaluate the physiological aspects of the NAVA over short periods of observation. All studies confirm improved patient-ventilator synchrony. Given that clinical experience suggests that certain patients might benefit from improved support, further studies are required to evaluate the clinical impact of NIV-NAVA on comfort, failure rates, and length of ventilatory support in critically ill children.

Keywords Non invasive ventilation $\cdot$ Neurally adjusted ventilatory assist $\cdot$ Pediatric critical care $\cdot$ Mechanical ventilation

\section{Introduction}

La détresse respiratoire est la première cause d'admission en réanimation pédiatrique. Plus de la moitié des patients admis dans une unité de soins intensifs pédiatriques nécessitent un support ventilatoire [1]. L'objectif de la ventilation est de permettre une décharge des muscles respiratoires tout en maintenant des échanges gazeux adéquats le temps que le processus pathologique responsable de la défaillance respiratoire se résolve. La ventilation invasive (VI) est une thérapie vitale et fut longtemps le seul support ventilatoire disponible en pédiatrie. La VI est associée à des effets secondaires délétères rapportés chez $40 \%$ des patients ventilés: complications mécaniques (sténose sous-glottique, pneumothorax), 
infectieuses (pneumonie associée au ventilateur) et lésions pulmonaires générées par la ventilation [2]. La ventilation non invasive (VNI), développée initialement chez l'adulte puis chez l'enfant, permet de supporter efficacement le patient [3-6] et de minimiser les complications liées à la VI $[7,8]$. La VNI est de plus en plus utilisée en réanimation pédiatrique [9-12], devenant le principal mode utilisé dans certaines pathologies [5]. En revanche, le taux d'échec de la VNI dans la population pédiatrique reste non négligeable, avec une fréquence rapportée entre 10 et $45 \%$ [10,13]. Les prédicteurs d'échec de la VNI incluent le jeune âge, le faible poids, la présence de pathologie chronique avant l'admission, les apnées, un ratio $\mathrm{SpO}_{2} / \mathrm{FiO}_{2}$ bas, l'absence d'amélioration du rythme cardiaque et de la fréquence respiratoire dans les premières heures et un score Pediatric Risk of Mortality (PRISM)-III à 24 h élevé $[14,15]$. La VNI peut être délivrée avec une simple pression positive continue (CPAP ou Continuous Positive Airway Pressure) ou une ventilation à deux niveaux de pressions, au cours de laquelle une interaction entre le patient et le ventilateur devient indispensable. L'asynchronie patient-ventilateur est très fréquente chez les enfants, en VI [16-18] comme en VNI [13,17,18]. L'asynchronie est une des causes reconnues d'échec de la VNI [21]. L'optimisation de la synchronie en pédiatrie représente un défi particulier pour les raisons suivantes : la présence de fuites à l'interface patient-masque favorisées par la variabilité de la taille des enfants rendant l'adaptation du masque difficile, la fréquence respiratoire élevée, les petits volumes courants [19,22]. Le mode NAVA (Neurally Adjusted Ventilatory Assist), dans lequel la ventilation est synchronisée avec le signal d'activité électrique du diaphragme, permet d'optimiser les interactions patient-respirateur, quelles que soient les fuites et les conditions mécaniques. Ce mode s'annonce donc particulièrement intéressant en contexte de VNI. L'objectif de cette revue est de préciser l'intérêt du mode NAVA en VNI pédiatrique et de fournir des repères pratiques pour l'utilisation de ce mode, à partir des données de la littérature et de notre expérience clinique.

\section{Principes du mode NAVA}

Le mode NAVA est un mode de ventilation asservi à la demande ventilatoire du patient, reflétée par son activité électrique du diaphragme (AEdi). L'AEdi est mesurée en continu à l'aide d'une sonde gastrique munie d'un faisceau de microélectrodes et d'un algorithme de traitement de signal validé, permettant de s'affranchir des mouvements du diaphragme par rapport aux électrodes et des artefacts provenant d'autres activités musculaires [23]. L'AEdi est un bon reflet de la « demande » ventilatoire des centres respiratoires du patient [24]. Tel qu'illustré sur la copie d'écran du ventilateur (Fig. 1), le ventilateur déclenche une assis-

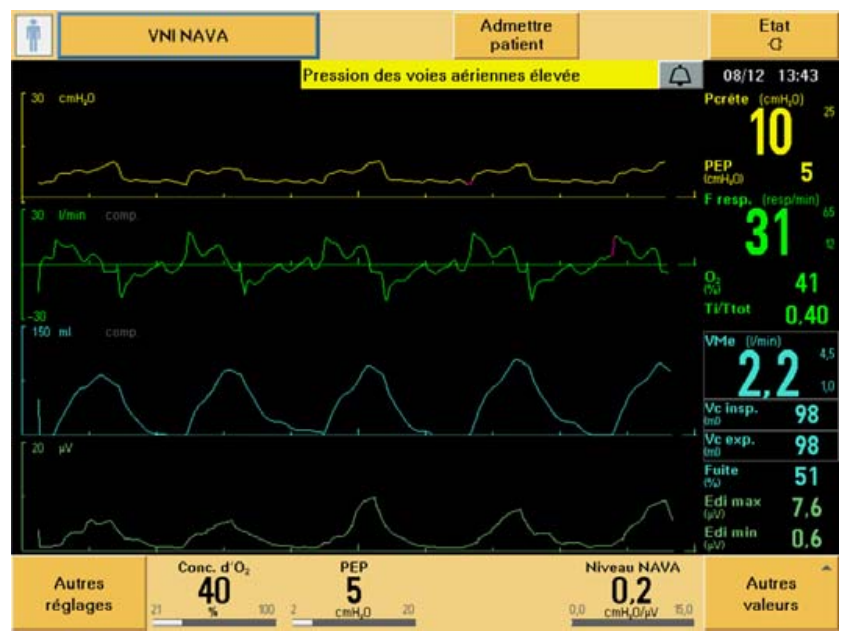

Fig. 1 Copie d'écran du ventilateur en mode VNI-NAVA

tance dès la détection d'une activation électrique du diaphragme, et cette assistance s'interrompt lorsque l'AEdi diminue à $70 \%$ de son pic. En outre, le support inspiratoire délivré est proportionnel à l'AEdi afin de permettre l'adaptation du support à la demande du patient [25]. Le niveau de l'aide inspiratoire $\left(\mathrm{cmH}_{2} \mathrm{O}\right)$ délivré est obtenu en multipliant le niveau NAVA $\left(\mathrm{cm} \mathrm{H}_{2} \mathrm{O} . \mu \mathrm{V}^{-1}\right)$ réglé par le niveau d'AEdi $(\mu \mathrm{V})$ mesurée. L'inspiration peut être déclenchée par le signal AEdi ou par le trigger pneumatique classique selon le système « premier arrivé premier servi ».

\section{Importance de l'asynchronie patient- ventilateur}

L'asynchronie patient-ventilateur est définie comme un décalage entre l'inspiration du patient et l'insufflation du ventilateur. La synchronisation adéquate entre le patient et le ventilateur permet d'améliorer le confort du patient, de réduire son travail respiratoire et de diminuer la durée de support ventilatoire chez l'adulte $[19,26]$. Afin de quantifier ce phénomène, l'index d'asynchronie (IA) est fréquemment utilisé, représentant le nombre de cycles asynchrones par minute en pourcentage du nombre total de cycles délivrés par le ventilateur. Un seuil de $10 \%$ est généralement considéré pour définir l'asynchronie sévère. Il faut noter que l'IA ne prend pas en compte les délais de réponse d'activation et inactivation du support par rapport à la demande du patient.

Les méthodes de référence pour déterminer de manière précise l'asynchronie nécessitent des mesures physiologiques invasives (pression œsophagienne ou électromyogramme du diaphragme), rarement utilisées en pratique clinique. Un premier intérêt notable du mode NAVA est la possibilité de détecter facilement les asynchronies, grâce à la visualisation simultanée des tracés d'AEdi, de pression 
et de volume. Plusieurs types d'asynchronie peuvent être observés chez l'enfant.

Les efforts inefficaces sont définis comme des efforts inspiratoires du patient non assistés par le ventilateur. Ce type d'asynchronie est très fréquent en pédiatrie en raison des faibles volumes et débits générés, et de la sensibilité insuffisante des triggers des respirateurs. Ces efforts inefficaces sont également favorisés par les pathologies obstructives générant une pression positive intrinsèque (PEPi). En effet l'effort développé par le patient peut être insuffisant pour contrecarrer la PEPi et déclencher le trigger. Cela a été démontré chez l'adulte [27], et plusieurs pathologies pédiatriques s'accompagnent de PEPi (bronchiolite, dysplasie broncho-pulmonaire, asthme aigu grave...). Le système de déclenchement du mode NAVA étant indépendant des conditions mécaniques, il n'est pas influencé par la surcharge imposée par la PEPi. Par ailleurs, les efforts inefficaces sont aussi favorisés par la sur-assistance. Là encore, le mode NAVA a un intérêt théorique par son principe d'assistance proportionnelle qui limite le risque de sur-assistance par rétrocontrôle.

Les auto-déclenchements sont des cycles délivrés par le ventilateur en l'absence d'effort. Les principaux facteurs favorisant ce type d'asynchronie sont les fuites, les oscillations cardiaques [28], et une sensibilité excessive du trigger. Ce type d'asynchronie est fréquent en VNI pédiatrique du fait des fuites et des performances du trigger [20]. Le trigger neural du mode NAVA n'est pas affecté par l'importance des fuites et réduit ce type d'asynchronie. Les doubles déclenchements correspondent à deux cycles délivrés consécutivement pour un même effort inspiratoire. Ils surviennent généralement quand la demande du patient est importante et que le temps d'insufflation du ventilateur est trop court.

Les délais d'activation et d'inactivation de l'assistance constituent une part importante d'asynchronie en pédiatrie. Les temps inspiratoires et expiratoires des enfants étant courts, les délais sont proportionnellement particulièrement importants $[17,18]$. Les délais d'activation et d'inactivation peuvent être positifs ou négatifs selon la réponse tardive ou prématurée du ventilateur [17]. Le concept de synchronisation du mode NAVA a également un intérêt dans ce type d'asynchronie car le délai de réponse est diminué.

De multiples effets délétères de l'asynchronie patientventilateur en VI sont décrits chez l'adulte: moins bonne réduction du travail respiratoire, inconfort, troubles du sommeil, support ventilatoire prolongé [26,29]. En VNI, l'asynchronie est associée à une moins bonne oxygénation, une moins bonne décharge des muscle respiratoire [30] et à un inconfort [30-32]. Tous ces éléments sont des facteurs de risque d'échec de la VNI. Chez l'enfant, les asynchronies sont plus fréquentes, avec un IA de 25 à $60 \%$ en fonction du type de support et des études $[17,31,33]$. L'augmentation de la durée de ventilation liée à l'asynchronie n'a pas été démontrée en pédiatrie, mais une association avec la décharge du travail respiratoire a été mise en évidence [19]. Chez des enfants avec obstruction des voies aériennes hautes ventilés en VNI, Essouri et al. [19] retrouvent une proportion importante $(>50 \%)$ de cycles ventilatoires non détectés et des délais inspiratoires élevés de l'ordre de $300 \mathrm{~ms}$. Dans ce même travail, la décharge des muscles respiratoires était moins bonne en VNI à deux niveaux d'assistance par rapport à la CPAP seule.

\section{Données de la littérature}

\section{Les études sur la VNI-NAVA chez l'adulte}

Les travaux préliminaires sur des volontaires sains ont confirmé l'efficacité de la VNI-NAVA en termes de décharge des muscles respiratoires et l'amélioration du confort [34,35]. Quatre études ont évalué le mode VNI-NAVA chez des patients de réanimation adulte, avec des devis de type crossover, évaluant le mode NAVA sur de brèves durées (10 à 30 minutes) et sur un petit effectif de patients (voir le Tableau 1) $[30,32,36,37]$. Ces études confirment toutes que la VNI en mode NAVA est associée à une amélioration significative de la synchronisation patient-ventilateur. Tous les patients avaient un taux d'asynchronie $<10 \%$ et une absence d'effort inefficace en VNI-NAVA. Cette amélioration est supérieure à celle démontrée par l'utilisation de l'algorithme VNI. Une diminution significative du délai inspiratoire était aussi observée, sous le seuil de $150 \mathrm{~ms}$, qui semble cliniquement pertinent [38].

\section{Les études sur la VNI-NAVA en réanimation pédiatrique}

En ventilation invasive, plusieurs études ont confirmé que le mode NAVA améliore significativement toutes les formes d'asynchronie chez les enfants [13,15,37]. En revanche, il existe peu d'études pédiatriques sur la VNI-NAVA (Tableau 1).

Beck et al. [40] en 2009 ont montré la faisabilité de la VNI-NAVA chez les prématurés de petits poids, malgré des fuites importantes. Cette étude portait sur sept nouveau-nés ventilés en NAVA invasif puis, au décours de l'extubation, en VNI-NAVA (durée 20 minutes). La synchronie en mode VNI-NAVA restait aussi bonne qu'en ventilation invasive NAVA.

Vignaux et al. [13] ont publié récemment une étude de type cross-over chez six enfants de 5 à 27 mois nécessitant une VNI suite à une détresse respiratoire post-extubation. L'évaluation de la synchronisation a été réalisée sur trois modes ventilatoires randomisés : VNI en mode conventionnel, VNI conventionnelle ajustée pour obtenir un cyclage expiratoire optimal, et VNI-NAVA. L'optimisation de la 
Tableau 1 Résumé des études adultes et pédiatriques portant sur la VNI-NAVA

\begin{tabular}{|c|c|c|c|c|}
\hline Étude & Population & Devis, type VNI, durée & $\begin{array}{l}\text { Interface } \\
\text { VNI }\end{array}$ & $\begin{array}{l}\text { Évolution en NAVA Autres paramètres } \\
\text { des paramètres } \\
\text { d'asynchronie }\end{array}$ \\
\hline
\end{tabular}

\section{Études en réanimation adulte}

Cammarota 10 patien

$2011[34]$ post-extubation

Piquilloud 13 patients

2012 [35] de réanimation

Schmidt

17 patients post-

Bertrand 13 patients

2013 [30] de réanimation

\section{Études en réanimation pédiatrique}

$\begin{array}{lll}\text { Vignaux } & \text { Six enfants } & \text { Croisée randomisée } \\ 2013[13] & \text { postopératoires } & \text { AI } 15 \mathrm{~min} \\ & & \text { AI TE }-15 \% 15 \mathrm{~min} \\ & & \text { AI TE }+15 \% 15 \mathrm{~min} \\ & & \text { NAVA } 20 \mathrm{~min}\end{array}$

Ducharme- 12 enfants

Crevier de réanimation

2013 [39]
Croisée

AI 20 minutes

NAVA 20 minutes

AI 20 minutes

Croisée randomisée

AI 20 min

NAVA $20 \mathrm{~min}$

Croisée randomisée AI 10 min

AI VNI+ $10 \mathrm{~min}$

NAVA $10 \mathrm{~min}$

NAVA VNI+ $10 \mathrm{~min}$

Croisée randomisée

NAVA $30 \mathrm{~min}$

AI 30 min

\section{Casque}

(2)

(1)

Masque

facial

Masque

oronasal

Masque

facial

Canules

nasales

Masque

facial

Croisée CPAP ou AI 30 min

NAVA $60 \mathrm{~min}$

CPAP ou AI 30 min

nasal

ou facial
Diminution délais inspiratoires

Diminution efforts inefficaces

Diminution Index

Asynchronie

\section{Diminution délais} inspiratoires

Diminution

des cyclages

prématurés et tardifs

Diminution efforts

inefficaces

Diminution Index

Asynchronie

Diminution délais

inspiratoires

Diminution

des cyclages

prématurés et tardifs

Diminution double

déclenchements

Diminution Index

Asynchronie

Diminution délais

inspiratoires

Diminution délais

de cyclage tardifs

Diminution efforts

inefficaces

Diminution Index

Asynchronie

Diminution délais

inspiratoires

Pas de changement :

AEdi, FR, Vt

Diminution cyclages

prématurés

Diminution efforts

inefficaces

Diminution Index

Asynchronie

Masque Diminution délais

inspiratoires

Pas de changement :

Diminution efforts inefficaces

Pas de changement :

AEdi, FR, gaz artériel, ratio $\mathrm{paO}_{2} / \mathrm{FiO}_{2}$

Pas de changement :

AEdi, FR, Vt, gaz

artériel, ratio $\mathrm{paO}_{2} / \mathrm{FiO}_{2}$

Diminution Vt

Aucun changement :

AEdi, FR

Augmentation AEdi

Pas de changement : Vt, gaz artériel, ratio $\mathrm{paO}_{2} /$ $\mathrm{FiO}_{2}$

AEdi, FR

$\mathrm{AI}$ : aide inspiratoire ; NAVA : neurally adjusted ventilatory assist ; VNI+ : algorithme pour VNI ; TE : trigger expiratoire ; AEdi : activité électrique du diaphragme ; FR : fréquence respiratoire ; Vt : volume courant 
VNI conventionnelle permettait une diminution modérée et non significative de l'IA ( $40 \%$ versus $65 \%, \mathrm{p}=0,05$ ), alors que la VNI-NAVA entraînait une forte réduction de l'IA ( $2 \%$ vs $65 \%, p<0,001)$. Aucun patient en mode NAVA n'a présenté d'effort inefficace ou de délai de cyclage significatif. De plus, le délai inspiratoire était significativement plus court en NAVA (61 ms versus $149 \mathrm{~ms}, \mathrm{p}<0,05)$, permettant un support inspiratoire plus long. Le volume courant et la fréquence respiratoire n'étaient pas significativement modifiés.

Une étude récemment réalisée par notre équipe sur l'évaluation du mode VNI-NAVA chez 12 enfants confirme également la franche amélioration de la synchronisation en mode VNI-NAVA [41].

En résumé, les études publiées chez l'adulte comme chez l'enfant confirment toutes l'intérêt potentiel majeur du mode NAVA pour améliorer la synchronisation en VNI. Ce bénéfice clair est également observé dans la pratique clinique, tel qu'illustré dans la Figure 2. Cependant, le bénéfice clinique de cette synchronisation optimisée en termes de succès de la VNI, de taux d'intubation, de durée de ventilation ou de séjour, reste à démontrer. Ces bénéfices éventuels devront également être mis en balance avec le coût additionnel lié à l'équipement spécifique du ventilateur et aux sondes gastriques spéciales. Il faut noter qu'en VNI pédiatrique, la mise en place d'un cathéter œsophagien au cours de la VNI est quasi systématique et ne correspond donc pas à une augmentation de l'invasivité avec ce mode.
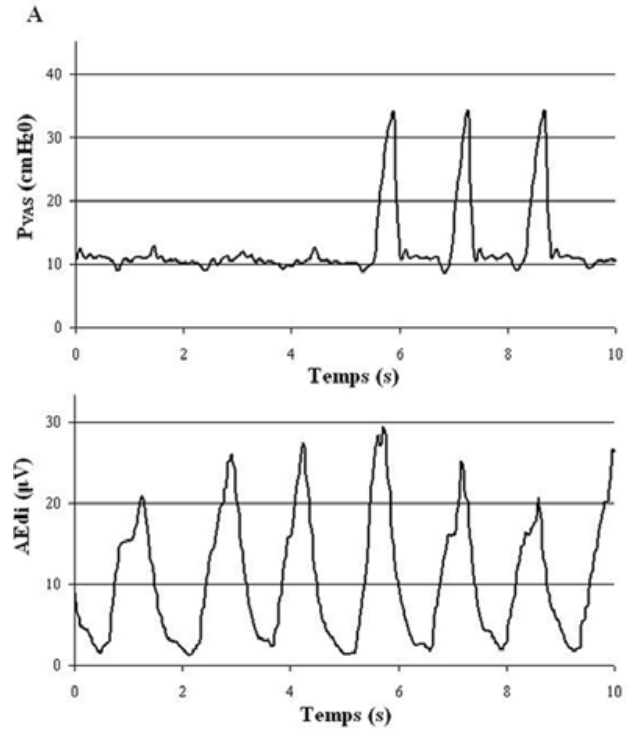

\section{La VNI-NAVA en pratique}

\section{Interfaces}

Plusieurs interfaces sont disponibles pour pratiquer la VNI en NAVA. Le choix de l'interface dépend de la sévérité de l'insuffisance respiratoire, du faciès du patient, de sa tolérance présumée et de son âge. Le masque naso-buccal ou facial permet une pression positive nasale et buccale, limitant les fuites par la bouche ouverte, et permettant d'assurer un support plus important. Ce type de masque est donc préférentiellement choisi chez les patients nécessitant un support important. Cependant, ces masques sont parfois moins bien tolérés que des interfaces nasales. Le masque nasal, les canules binasales et le tube nasopharyngé exposent à des fuites plus importantes mais elles semblent mieux tolérées, permettent la vocalisation, et limitent le risque d'inhalation lors de vomissements. Les fuites importantes avec ces interfaces ne perturbent pas la synchronisation en mode VNINAVA, mais la qualité de la transmission du support aux voies aériennes basses est plus incertaine. Aucune étude n'a rapporté de comparaison des interfaces en mode NAVA en pédiatrie. Il semblerait y avoir chez les patients adultes une tolérance accrue au masque nasal comparé au masque facial et aux lunettes nasales [42,43]. Les masque faciaux récemment développés sont particulièrement bien tolérés dans la pratique pédiatrique, et ils sembleraient plus efficaces que les autres interfaces $[42,43]$.
B
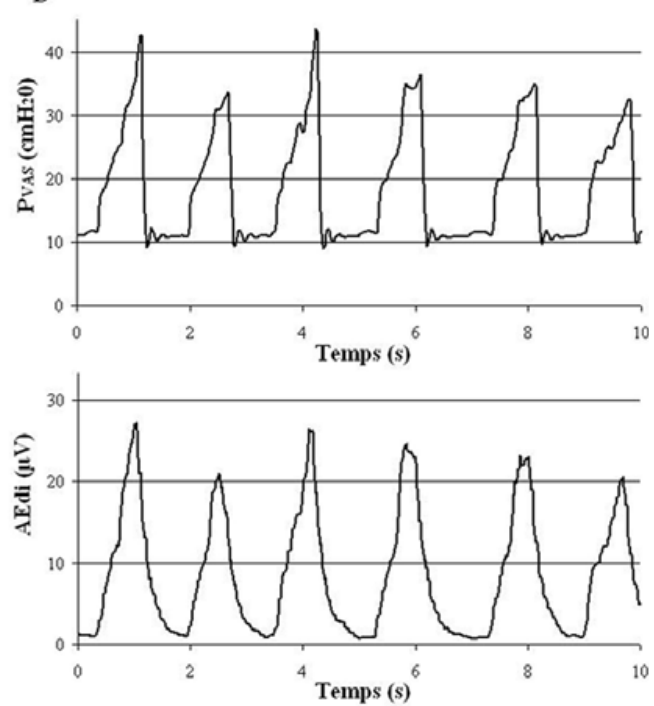

Fig. 2 Exemple représentatif de la synchronisation des tracés de pression des voies aériennes supérieures (PVAS) et de l'activité électrique du diaphragme (AEdi) d'un enfant sous ventilation non invasive en mode aide inspiratoire (panel A) et en mode NAVA (Neurally Adjusted Ventilatory Assist) (panel B). On observe la disparition des efforts inefficaces et la meilleure synchronisation et variabilité en mode NAVA 


\section{Sonde gastrique spécifique}

La sonde gastrique munie de microélectrodes pour la mesure de l'AEdi est disponible en taille $6 \mathrm{~F}, 8 \mathrm{~F}$, et $12 \mathrm{~F}$. La distance d'installation peut être estimée grâce à une formule fournie par le fabricant (à partir de la distance nez-oreille-xiphoïde), puis la position doit être vérifiée à l'aide de l'écran spécifique «NAVA catheter positioning » du Servo I. Cet écran affiche quatre dérivations rapportant l'activité ECG et diaphragmatique. Les trois signes nécessaires pour confirmer la bonne position sont : 1) la diminution de l'amplitude des complexes QRS au niveau distal ; 2) la présence des ondes $\mathrm{P}$ sur les deux premiers signaux avec atténuation en distalité ; et 3) la position du signal diaphragmatique bleu majoritairement dans les deux tracés centraux [44].

\section{Réglages}

\section{Le niveau NAVA}

En VNI tout comme en VI, la méthode idéale pour définir le niveau NAVA reste à déterminer. Dans la plupart des études mentionnées, le niveau NAVA était réglé pour obtenir une aide inspiratoire identique à celle délivrée en VNI conventionnelle. C'est certainement la technique la plus simple et la plus utilisée. Cependant, l'essentiel est de rapidement réévaluer le niveau NAVA après quelques minutes, car l'amplitude de l'AEdi change fréquemment après le passage en NAVA. Il faut donc s'assurer que le nouveau niveau de support généré est adéquat, notamment en fonction de la fréquence respiratoire, du volume courant et des efforts du patient (cliniques et reflétés par l'AEdi). L'adaptation du niveau NAVA pour cibler une valeur d'AEdi semble prometteuse, en visant soit des valeurs «normales » d'AEdi (qui restent à définir), soit un pourcentage de l'AEdi mesuré au cours d'une épreuve standardisée [45]. Une autre méthode a été récemment décrite par Chiew et al. [46] chez 12 patients adultes de réanimation. Le niveau NAVA était d'abord réglé afin d'obtenir le même degré d'aide inspiratoire qu'en VNI conventionnelle, puis modifié à $50 \%$ puis $150 \%$ de cette valeur initiale. À chaque palier, la relation entre le volume courant et l'intégrale de l'AEdi (reflet de la demande) était évaluée. Le Range90, correspondant à l'intervalle entre les $5^{\mathrm{e}}$ et $95^{\mathrm{e}}$ percentiles de ce ratio $\mathrm{Vt} / \triangle \mathrm{AEdi}$, était utilisé pour comparer les différents niveaux NAVA. Plus le Range 90 est élevé, plus le support respiratoire est inconsistant et variable, reflétant une moins bonne adéquation entre le volume courant et la demande du patient. Dans cette série de 12 patients, le seuil de NAVA à $50 \%$ était considéré comme optimal. Par contre, les données individuelles étaient très éparses, suggérant que le niveau NAVA doit être estimé individuellement. Cette approche pourrait être évaluée chez l'enfant.

\section{Autres réglages}

La pression positive expiratoire (PEEP) et la $\mathrm{FiO}_{2}$ sont ajustées de façon similaire en VNI-NAVA et en VNI conventionnelle. Lors d'une transition du mode CPAP au mode NAVA, l'ajout de l'assistance inspiratoire entraîne une hausse de la pression moyenne des voies aériennes, et une diminution de la PEEP peut être nécessaire. L'activité tonique du diaphragme (AEdi persistant pendant l'expiration) permet le maintien du volume pulmonaire chez les nourrissons [33]. Une augmentation inhabituelle de l'AEdi tonique peut donc refléter des efforts importants pour maintenir ce volume pulmonaire ; devant ce signal, la pertinence d'une augmentation du niveau de PEEP peut donc être considérée [33].

Tel que discuté plus haut, le déclenchement de l'aide inspiratoire peut se faire en trigger AEdi ou en trigger pneumatique. Le trigger neural est réglé par défaut à $0,5 \mu \mathrm{V}$, et il est rarement nécessaire de le modifier. Le trigger pneumatique et le seuil de cyclage ne sont pas réglables en mode VNI. Une ventilation d'apnée en pression contrôlée doit être prescrite.

Un paramètre essentiel en mode NAVA est l'alarme de pression maximale, qui limite la pression maximale de support à $5 \mathrm{cmH}_{2} \mathrm{O}$ sous ce niveau d'alarme. Ce paramètre est crucial, car des surpressions importantes pourraient survenir chez les patients dont l'AEdi est très élevée.

\section{Surveillance}

La VNI-NAVA nécessite une surveillance rapprochée similaire aux autres modes de VNI. Une particularité de ce mode est la surveillance régulière de la position adéquate du cathéter à l'aide de l'écran de positionnement et à l'aide des repères inscrits sur le cathéter.

En plus de permettre la synchronisation, l'AEdi est un bon indicateur de l'évolution de la demande et donc probablement du travail respiratoire du patient. Il est donc important de suivre l'évolution de ce paramètre. Un niveau d'AEdi faible $(<3 \mu \mathrm{V})$ peut indiquer une assistance trop importante du ventilateur, une sédation significative ou une atteinte centrale de la commande respiratoire. Au contraire, une AEdi élevée suggère le plus souvent un travail respiratoire important du patient, ou parfois une inefficacité du couplage neuromusculaire qui entraîne une élévation de la commande centrale (ex : pathologie neuromusculaire) [44]. Les valeurs normales de l'AEdi ne sont pas connues, mais l'évolution de l'AEdi dans le temps est un nouvel outil particulièrement utile pour le suivi d'un même patient.

\section{Situations particulières et indications préférentielles}

La VNI-NAVA présente un intérêt clinique potentiel important chez les patients pour lesquels une bonne synchronisation est essentielle. La Figure 3 présente un algorithme qui 


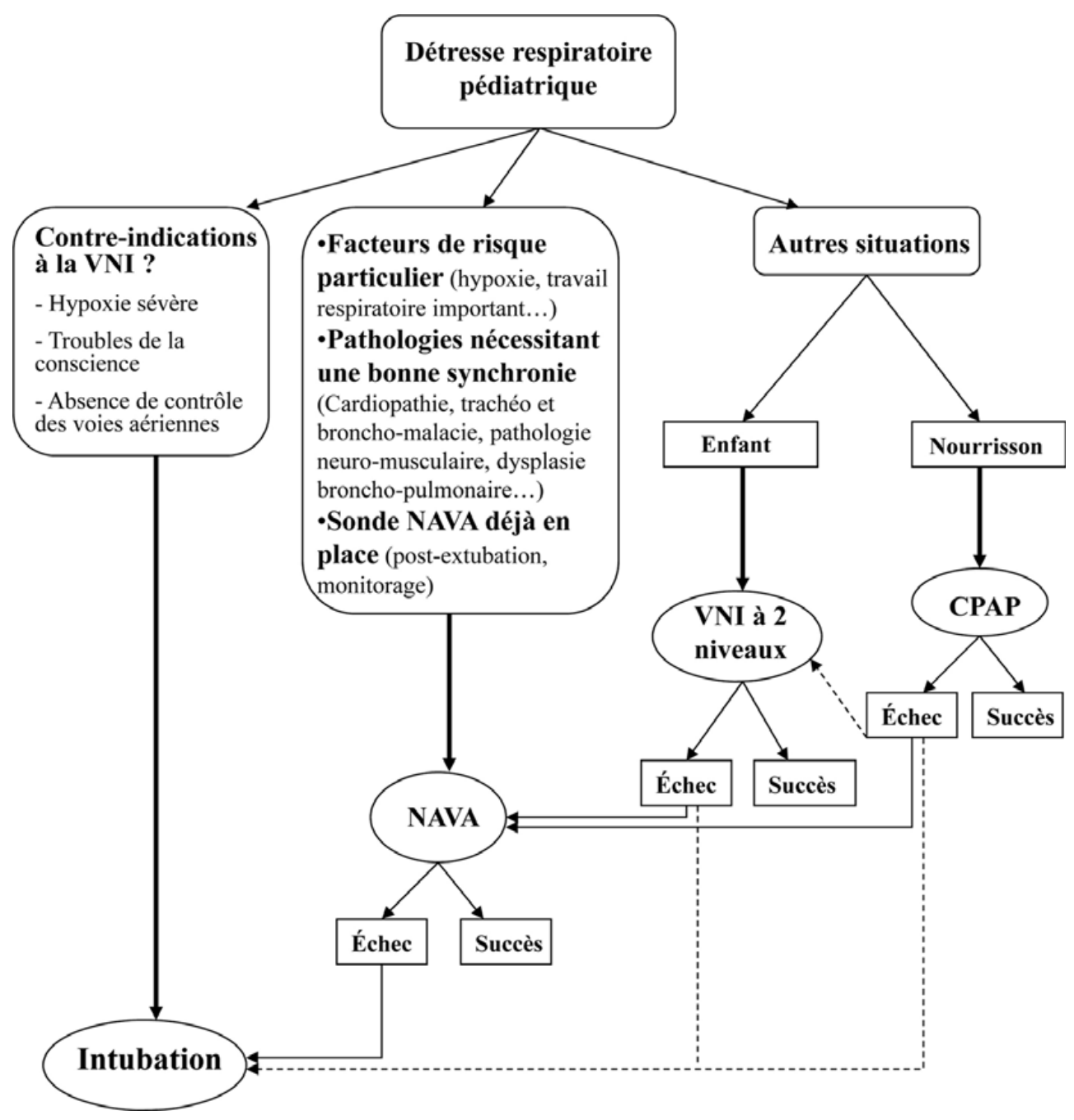

Fig. 3 Algorithme décrivant notre pratique pour le choix du mode de ventilation non invasive en réanimation pédiatrique. Cet algorithme est indicatif et reflète les situations les plus fréquentes, mais d'autres choix sont régulièrement faits en fonction des conditions particulières. VNI : ventilation non invasive ; CPAP : Continuous Positive Airway Pressure; NAVA : Neurally Adjusted Ventilatory Assist

schématise notre pratique concernant le choix du mode de VNI en réanimation pédiatrique. À ce jour, aucune étude n'a été effectuée pour évaluer les indications préférentielles de ce mode. Cette population peut être identifiée selon l'expérience clinique et certaines études sur les facteurs de risque d'échec de la VNI. La VNI-NAVA implique des coûts supplémentaires liés au cathéter nasogastrique, il faut donc choisir avec soins les patients éligibles. Par exemple, dans la bronchiolite, le succès élevé de la VNI conventionnelle ne justifie pas l'implémentation de ce support en première intention. En revanche, les patients présentant un travail respiratoire important et/ou une hypoxémie associée (détresse respiratoire sévère, syndrome de détresse respiratoire aiguë, mucoviscidose) ont un taux important d'échec à la VNI [15]; il importe donc de fournir dans ces conditions précises le meilleur support possible et l'amélioration de la synchronisation grâce au mode NAVA pourrait avoir un impact clinique significatif dans cette population sélectionnée. Chez les patients avec obstruction des voies aériennes supérieures (trachéomalacie, bronchomalacie), la mauvaise synchronisation patient-ventilateur a un impact négatif sur le travail respiratoire et pourrait particulièrement bénéficier du mode NAVA. Les patients avec paralysie unilatérale du diaphragme, dysplasie bronchopulmonaire, cardiopathies, maladies neuromusculaires, tolèrent également mal la mauvaise synchronisation et pourraient eux aussi bénéficier de l'amélioration de la synchronisation en mode NAVA. Toutefois, la supériorité clinique de la VNI-NAVA dans ces indications reste à confirmer.

\section{Problèmes potentiels}

Certains problèmes spécifiques à la VNI-NAVA sont à connaître et à identifier. Les fuites excessives ne causent 
pas d'anomalies du déclenchement en NAVA, mais peuvent déclencher une aide inspiratoire en trigger pneumatique. Tout comme en VNI conventionnelle, les fuites altèrent la transmission efficace de l'aide inspiratoire aux voies aériennes basses. La présence d'apnées centrales expose au risque d'hypoventilation, comme en aide inspiratoire. Une perte de signal AEdi peut survenir secondairement à un déplacement du cathéter, une sédation importante, ou une sur-assistance par le ventilateur; un relais en VNI conventionnel intervient, mais il peut être moins bien toléré. Il convient de bien régler les modalités de passage en VNI AI et de bien régler le niveau d'AI souhaité en cas de perte de signal AEdi.

Enfin, comme dans toute situation de VNI, il faut savoir reconnaître rapidement le patient qui nécessite une intubation. L'amélioration de la qualité du support non invasif avec le mode NAVA peut permettre d'éviter certains échecs de la VNI, mais il est important de réaliser qu'en l'absence d'amélioration significative après les premières une à deux heures de support, le taux d'échec de VNI est important et qu'une intubation trop tardive peut être associée à une morbidité plus élevée.

\section{Conclusion}

Les études actuelles sur la VNI en NAVA sont encore essentiellement d'ordre physiologique et à court terme. Elles démontrent la faisabilité de ce support et l'amélioration significative des tous les types d'asynchronie patient-ventilateur en VNI-NAVA, chez l'adulte comme chez l'enfant. Ces données sont encourageantes et supportent l'utilisation future de la VNI-NAVA. Cependant, le bénéfice de cette synchronie améliorée reste imprécis et les évidences actuelles ne permettent pas de confirmer l'intérêt de la VNI-NAVA sur l'évolution clinique des patients. De prochaines études devraient permettre l'évaluation clinique de la VNI-NAVA en termes de confort, durée de ventilation, taux d'échec de la VNI, ainsi que préciser les bonnes indications.

Conflit d'intérêt : L. Ducharme-Crevier, S. Essouri, A. Larouche et G. Emeriaud déclarent ne pas avoir de conflit d'intérêt.

\section{Références}

1. Newth CJL, Venkataraman S, Willson DF, et al (2009) Weaning and extubation readiness in pediatric patients. Pediatr Crit Care Med J Soc Crit Care Med World Fed Pediatr Intensive Crit Care Soc 10:1-11

2. Principi T, Fraser DD, Morrison GC, et al (2011) Complications of mechanical ventilation in the pediatric population. Pediatr Pulmonol 46:452-7
3. L'Her E, Deye N, Lellouche F, et al (2005) Physiologic effects of noninvasive ventilation during acute lung injury. Am J Respir Crit Care Med 172:1112-8

4. Essouri S, Durand P, Chevret L, et al (2008) Physiological effects of noninvasive positive ventilation during acute moderate hypercapnic respiratory insufficiency in children. Intensive Care Med 34:2248-55

5. Yanez LJ, Yunge M, Emilfork M, et al (2008) A prospective, randomized, controlled trial of noninvasive ventilation in pediatric acute respiratory failure. Pediatr Crit Care Med 9:484-9

6. Cambonie G, Milési C, Jaber S, et al (2008) Nasal continuous positive airway pressure decreases respiratory muscles overload in young infants with severe acute viral bronchiolitis. Intensive Care Med 34:1865-72

7. Antonelli M, Conti G, Rocco M, et al (1998) A Comparison of Noninvasive Positive-Pressure Ventilation and Conventional Mechanical Ventilation in Patients with Acute Respiratory Failure. N Engl J Med 339:429-35

8. Girou E, Schortgen F, Delclaux C, et al (2000) Association of noninvasive ventilation with nosocomial infections and survival in critically ill patients. JAMA J Am Med Assoc284:2361-7

9. Essouri S, Chevret L, Durand P, et al (2006) Noninvasive positive pressure ventilation: Five years of experience in a pediatric intensive care unit. Pediatr Crit Care Med 7:329-34

10. Najaf-Zadeh A, Leclerc F (2011) Noninvasive positive pressure ventilation for acute respiratory failure in children: a concise review. Ann Intensive Care 1:15

11. Farias JA, Fernández A, Monteverde E, et al (2012) Mechanical ventilation in pediatric intensive care units during the season for acute lower respiratory infection: a multicenter study. Pediatr Crit Care Med J Soc Crit Care Med World Fed Pediatr Intensive Crit Care Soc 13:158-64

12. Ganu SS, Gautam A, Wilkins B, Egan J (2012) Increase in use of non invasive ventilation for infants with severe bronchiolitis is associated with decline in intubation rates over a decade. Intensive Care Med 38:1177-83

13. Vignaux LP, Grazioli S, Piquilloud L, et al (2013) Patientventilator asynchrony during noninvasive pressure support ventilation and neurally adjusted ventilatory assist in infants and children. Pediatr Crit Care Med 14:e357-64

14. Mayordomo-Colunga J, Medina A, Rey C, et al (2009) Success and failure predictors of non invasive ventilation in acute bronchiolitis. An Pediatría Barc Spain 70:34-9

15. Mayordomo-Colunga J, Pons M, López Y, et al (2013) Predicting non invasive ventilation failure in children from the $\mathrm{SpO}_{2} / \mathrm{FiO}_{2}$ (SF) ratio. Intensive Care Med 39:1095-103

16. Vignaux LM, Grazioli S, Piquilloud L, et al (2013) Optimizing Patient-Ventilator Synchrony During Invasive Ventilator Assist in Children and Infants Remains a Difficult Task. Pediatr Crit Care Med 14:316-25

17. Bordessoule A, Emeriaud G, Morneau S, et al (2012) Neurally adjusted ventilatory assist improves patient-ventilator interaction in infants as compared with conventional ventilation. Pediatr Res 72:194-202

18. Beck J, Tucci M, Emeriaud G, et al (2004) Prolonged Neural Expiratory Time Induced by Mechanical Ventilation in Infants. Pediatr Res 55:747-54

19. Essouri S, Nicot F, Clément A, et al (2005) Noninvasive positive pressure ventilation in infants with upper airway obstruction: comparison of continuous and bilevel positive pressure. Intensive Care Med 31:574-80

20. Vignaux L, Vargas F, Roeseler J, et al (2009) Patient-ventilator asynchrony during non invasive ventilation for acute respiratory failure: a multicenter study. Intensive Care Med 35:840-6

21. Hess DR (2011) Patient-ventilator interaction during noninvasive ventilation. Respir Care 56:153-65 
22. Moerer O, Barwing J, Quintel M (2008) Neurally adjusted ventilatory assist (NAVA). A new mode of assisted mechanical ventilation. Anaesthesist 57:998-1005

23. Sinderby CA, Beck JC, Lindström LH, Grassino AE (1997) Enhancement of signal quality in esophageal recordings of diaphragm EMG. J Appl Physiol Bethesda Md 82:1370-7

24. Lourenço RV, Cherniack NS, Malm JR, Fishman AP (1966) Nervous output from the respiratory center during obstructed breathing. J Appl Physiol 21:527-33

25. Sinderby C, Navalesi P, Beck J, et al (1999) Neural control of mechanical ventilation in respiratory failure. Nat Med 5:1433-6

26. Thille A, Rodriguez P, Cabello B, et al (2006) Patient-ventilator asynchrony during assisted mechanical ventilation. Intensive Care Med 32:1515-22

27. Nava S, Bruschi C, Fracchia C, et al (1997) Patient-ventilator interaction and inspiratory effort during pressure support ventilation in patients with different pathologies. Eur Respir J 10:177-83

28. Imanaka H, Nishimura M, Takeuchi M, et al (2000) Autotriggering caused by cardiogenic oscillation during flow-triggered mechanical ventilation. Crit Care Med 28:402-7

29. Pierson DJ (2011) Patient-Ventilator Interaction. Respir Care 56:214-28

30. Schmidt M, Dres M, Raux M, et al (2012) Neurally adjusted ventilatory assist improves patient-ventilator interaction during postextubation prophylactic noninvasive ventilation. Crit Care Med 40:1738-44

31. Vignaux L, Tassaux D, Carteaux G, et al (2010) Performance of noninvasive ventilation algorithms on ICU ventilators during pressure support: a clinical study. Intensive Care Med 36:2053-9

32. Bertrand PM, Futier E, Coisel Y, et al (2013) Neurally adjusted ventilatory assist vs pressure support ventilation for noninvasive ventilation during acute respiratory failure: a crossover physiologic study. Chest 143:30-6

33. Emeriaud G, Beck J, Tucci M, et al (2006) Diaphragm electrical activity during expiration in mechanically ventilated infants. Pediatr Res 59:705-10

34. Sinderby C, Beck J, Spahija J, et al (2007) Inspiratory muscle unloading by neurally adjusted ventilatory assist during maximal inspiratory efforts in healthy subjects. CHEST J 131:711-7

35. Moerer O, Beck J, Brander L, et al (2008) Subject-ventilator synchrony during neural versus pneumatically triggered non invasive helmet ventilation. Intensive Care Med 34:1615-23
36. Cammarota G, Olivieri C, Costa R, et al (2011) Noninvasive ventilation through a helmet in postextubation hypoxemic patients: physiologic comparison between neurally adjusted ventilatory assist and pressure support ventilation. Intensive Care Med 37:1943-50

37. Piquilloud L, Tassaux D, Bialais E, et al (2012) Neurally adjusted ventilatory assist (NAVA) improves patient-ventilator interaction during non invasive ventilation delivered by face mask. Intensive Care Med 38:1624-31

38. Whitelaw WA, Derenne JP, Milic-Emili J (1975) Occlusion pressure as a measure of respiratory center output in conscious man. Respir Physiol 23:181-99

39. Breatnach C, Conlon NP, Stack M, et al (2010) A prospective crossover comparison of neurally adjusted ventilatory assist and pressure-support ventilation in a pediatric and neonatal intensive care unit population. Pediatr Crit Care Med 11:7-11

40. Beck J, Reilly M, Grasselli G, et al (2009) Patient-ventilator Interaction during Neurally Adjusted Ventilatory Assist in Very Low Birth Weight Infants. Pediatr Res 65:663-8

41. Ducharme-Crevier L, Emeriaud G, Beck J, Jouvet P (2012) Feasibility of neurally adjusted ventilatory assist (NAVA) during pediatric non invasive ventilation. (abstract). Crit Care Med 40:1-328

42. Girault C, Briel A, Benichou J, et al (2009) Interface strategy during noninvasive positive pressure ventilation for hypercapnic acute respiratory failure. Crit Care Med 37:124-31

43. Navalesi P, Costa R (2003) New modes of mechanical ventilation: proportional assist ventilation, neurally adjusted ventilatory assist, and fractal ventilation. Miscellaneous Article. Curr Opin Crit Care 9:51-8

44. Ducharme-Crevier L, Du Pont-Thibodeau G, Emeriaud G (2013) Interest of monitoring diaphragmatic electrical activity in the pediatric intensive care unit. Crit Care Res Pract 2013:384210

45. Rozé H, Lafrikh A, Perrier V, et al (2011) Daily titration of neurally adjusted ventilatory assist using the diaphragm electrical activity. Intensive Care Med 37:1087-94

46. Chiew YS, Chase JG, Lambermont B, et al (2013) Effects of Neurally Adjusted Ventilatory Assist (NAVA) levels in non invasive ventilated patients: titrating NAVA levels with electric diaphragmatic activity and tidal volume matching. Biomed Eng OnLine 12:61 